\title{
Case: Bacillus Calmette-Guerin (BCG)-induced Reiter syndrome with an attempt at repeat BCG induction
}

Douglas C. Cheung; Alexandra L. Millman; Robert J. Hamilton

University of Toronto, Department of Surgery, Division of Urology, University Health Network, Toronto, ON, Canada

Cite as: Can Urol Assoc J 2017 Dec 1; Epub ahead of print. http://dx.doi.org/10.5489/cuaj.4759

Published online December 1, 2017

$* * *$

\section{Introduction}

Non-muscle-invasive bladder cancer (NMIBC) is a significant cause of morbidity worldwide, requiring routine surveillance, resection/fulguration, and even cystectomy when refractory. ${ }^{1}$ In addition to resection, Bacillus Calmette-Guerin (BCG) intravesical immunotherapy is welldescribed with level 1 evidence demonstrating benefit in preventing recurrence and progression of NMIBC; ;,3 however, BCG treatment is not benign and carries risk of significant adverse effects. ${ }^{4,5}$

In particular, BCG-induced Reiter syndrome - a symptom complex consisting of urethritis, reactive arthritis, and conjunctivitis - is an extremely rare entity in the literature. ${ }^{6,7}$ In reported cases, BCG discontinuation and alternative methods to manage the bladder cancer were required. Unfortunately, these case reports do not document the long-term followup and clinical outcomes of these patients.

We present a case of BCG-induced Reiter syndrome with 19-year followup, and the first instance of BCG re-induction in this cohort of patients. This was attempted given the substantial duration of disease control following the initial treatment.

\section{Case report}

Our patient initially presented as a 49-year-old gentleman referred from the community with a filling defect in the right renal pelvis. His ECOG performance status was 0 . He is a non-smoker and had no risk factors for urothelial carcinoma.

In 1997, he underwent an open right nephroureterectomy with bladder cuff excision, demonstrating high-grade papillary urothelial cell carcinoma (T3N1M0) with carcinoma in-situ (CIS). There was extensive squamous differentiation and focal lymphovascular invasion. The ureter was involved with multifocal CIS, however, the distal margin was negative. Pathology demonstrated hilar node involvement despite negative pre-operative abdominal-pelvis CT and chest X-ray. His post-operative course was uncomplicated and he received four cycles of MVAC 
adjuvant chemotherapy (methotrexate/vinblastine/doxorubicin/cisplatin) given his node-positive disease. No adjuvant intravesical chemotherapy was given.

Unfortunately during surveillance, he subsequently developed gross hematuria and positive cytology. Eleven months after his nephroureterectomy, he required transurethral resection of bladder tumour (TURBT), demonstrating CIS. He was started on full dose intravesical BCG induction, with a plan for three-years maintenance.

After his second induction dose of BCG, he had a febrile episode and his third instillation was given at 1/10 dose, however, he resumed full dose for doses 4, 5 and 6 . He received his first maintenance without issue. However, following his 6-month maintenance, he developed prostatitis (elevated PSA and a firm nodule), which was managed with amoxicillin and ciprofloxacin. Transrectal ultrasound-guided biopsy revealed granulomatous disease. His BCG maintenance continued as scheduled. During his 18-month maintenance, two doses were held due to UTI.

His most significant reaction to BCG presented during his 30-month maintenance. After his second instillation, he developed fever, and severe cystitis. His third BCG instillation was held and he was started on a 3-month course of isoniazid. Shortly afterwards, he presented with lower limb swelling and pain. He was seen by Rheumatology and diagnosed with BCG induced Reiter syndrome. His constellation of symptoms included fever, bilateral lower limb arthritis, urethritis, and conjunctivitis. His reactive arthritis was managed with joint space steroid injections, daily oral prednisone (15mg then tapered), and rofecoxib prn. His final round of maintenance BCG, was not given.

On surveillance, he remained recurrence free for fifteen years until a positive cytology November 2014. In January 2016, a CT urogram demonstrated suspicious left upper pole calyceal thickening. Ureteroscopy revealed a urothelial tumour, which was biopsied (TaLG) and ablated. Additionally, he had a bladder recurrence with TURBT revealing T1HG and CIS and immediate re-resection for TaHG and CIS. Imaging (CXR and abdominal-pelvic CT scan) showed no definitive metastatic disease.

Notably, the patient did not wish to undergo cystectomy, and given his excellent prior response to BCG and significant time-lapse since his last treatment, a decision at multidisciplinary tumour boards was made to re-induce with full-dose intravesical BCG despite his previous BCG-induced Reiter syndrome.

He tolerated the first two instillations well, with minor complaints of dysuria and hematuria. However, following his third dose, he developed worsening dysuria, fever, and right knee swelling. He presented to the Emergency Department twice, and after consultation with his rheumatologist, he continued with full dose BCG and medical management of his reactive arthritis with steroid injections and oral NSAIDS. His fourth instillation of BCG was delayed by two-weeks due to fever, polyarthritis (left knee, bilateral ankles, left wrist, left TMZ, diffuse enthesitis), and episcleritis. Again, the decision was to continue NSAIDS and receive the final induction doses as scheduled. After completing his induction BCG, Rheumatology took a more 
active approach to managing his arthritis and he was started on oral sulfasalazine (1500mg BID) and prednisone (7.5mg daily). On this regimen, his symptoms completely resolved.

\section{Discussion}

Repeat induction with BCG following significant intolerance, especially BCG-induced Reiter syndrome, has not been previously described. We document our experience here over 19 years.

BCG-induced Reiter syndrome involving the full triad of reactive arthritis remains a rare entity. Although the pathophysiology is not completely understood, rheumatologic disease and its resultant significant functional impairment, has been well associated with Mycobacterium. ${ }^{\text {, } 8}$ One possible explanation following induction is its mediation through an immunologic response involving lymphocytic T cell response in the unintended tissues (i.e. synovial fluid). ${ }^{9,10} \mathrm{In}$ previous cases of Reiter syndrome, BCG has been stopped prematurely with adjunctive supportive therapy. ${ }^{11}$ Treatment has been targeted at managing: i) the symptomatology with nonsteroidal anti-inflammatory drugs (NSAIDs) for pain control; ii) the etiology with isoniazid (INH) and rifampin therapy; and iii) the immunologic response with disease modifying therapy including corticosteroids. ${ }^{10,12}$ However, in each case, little is known about long term follow-up and subsequent oncologic management. ${ }^{6}$

Although adjunctive therapies offer some mediation, the literature recommends halting BCG induction for uncontrolled symptomatology. ${ }^{9,}{ }^{11}$ As a result, no studies exist to guide attempted BCG re-induction following prior adverse events. We proceeded with this approach following multi-disciplinary tumor boards consensus, Rheumatology consult, and thorough shared decision-making discussion with our patient weighing the benefits of BCG induction (long duration 16-year disease-free interval) versus the possibility of significant adverse effect. Dose reduction suggestions by rheumatology included $1 / 10^{\text {th }}$ or even $1 / 100^{\text {th }}$ dosing. Further, there is a paucity of evidence to support treatment efficacy at such low doses; even studies of $1 / 3^{\text {rd }}$ and $1 / 6^{\text {th }}$ dosing show decreased efficacy. ${ }^{13-15}$

Our patient had a significant reaction following BCG re-induction. Despite this, the symptoms were managed expectantly with complete resolution and he was able to tolerate all induction treatments at full dose, albeit requiring a two-week break between his third and fourth instillations. As a result, judicious BCG re-induction is certainly possible despite previous severe reaction to BCG, but requires caution and close surveillance. We advocate for Rheumatology involvement for supportive management and dose assessment/reduction as necessary. Discussion with the patient should employ shared decision-making models, tailored to a specific patient's presentation and prior response, to clearly delineate risk versus benefit of treatment. Although more research is required, our study demonstrates that repeat induction is an option in the correct patient setting.

\section{Conclusion}

BCG re-induction remains a possibility despite prior significant reaction. We document our experience with the management of repeat BCG induction following BCG-induced Reiter syndrome in the primary induction series. In carefully selected patients and following a shared 
discussion model, we recommend active Rheumatology involvement, consideration for dosereduced therapy, adjuvant supportive measures, and close monitoring. Further research is required to delineate the optimal management of these patients. 


\section{References}

1. Kaufman, D. S., W. U. Shipley and A. S. Feldman. Bladder cancer. Lancet 2009;374:239-49. http://www.ncbi.nlm.nih.gov/pubmed/19520422

2. Lamm, D. L. Preventing progression and improving survival with BCG maintenance. European urology 2000;37 Suppl 1:9-

15. http://www.ncbi.nlm.nih.gov/pubmed/10575266

3. Sylvester, R. J., M. A. van der and D. L. Lamm. Intravesical bacillus Calmette-Guerin reduces the risk of progression in patients with superficial bladder cancer: a meta-analysis of the published results of randomized clinical trials. The Journal of urology 2002;168:1964-70. http://www.ncbi.nlm.nih.gov/pubmed/12394686

4. Fuge, O., N. Vasdev, P. Allchorne, et al. Immunotherapy for bladder cancer. Research and reports in urology 2015;7:65-79. http://www.ncbi.nlm.nih.gov/pubmed/26000263

5. Shelley, M. D., M. D. Mason and H. Kynaston. Intravesical therapy for superficial bladder cancer: a systematic review of randomised trials and meta-analyses. Cancer treatment reviews 2010;36:195-205. http://www.ncbi.nlm.nih.gov/pubmed/20079574

6. Taniguchi, Y., H. Nishikawa, T. Karashima, et al. Frequency of reactive arthritis, uveitis, and conjunctivitis in Japanese patients with bladder cancer following intravesical BCG therapy: A 20-year, two-centre retrospective study. Joint, bone, spine : revue du rhumatisme 2016; http://www.ncbi.nlm.nih.gov/pubmed/27825571

7. Lamm, D. L., P. M. van der Meijden, A. Morales, et al. Incidence and treatment of complications of bacillus Calmette-Guerin intravesical therapy in superficial bladder cancer. The Journal of urology 1992;147:596-

600. http://www.ncbi.nlm.nih.gov/pubmed/1538436

8. Franco-Paredes, C., A. Diaz-Borjon, M. A. Senger, et al. The ever-expanding association between rheumatologic diseases and tuberculosis. The American journal of medicine 2006;119:470-7. http://www.ncbi.nlm.nih.gov/pubmed/16750957

9. Tinazzi, E., V. Ficarra, S. Simeoni, et al. Reactive arthritis following BCG immunotherapy for urinary bladder carcinoma: a systematic review. Rheumatology international 2006;26:481-8. http://www.ncbi.nlm.nih.gov/pubmed/16220289

10. Tishler, M. and Y. Shoenfeld. BCG immunotherapy--from pathophysiology to clinical practice. Expert opinion on drug safety 2006;5:225-

9. http://www.ncbi.nlm.nih.gov/pubmed/16503744

11. Ben Abdelghani, K., A. Fazaa, L. Souabni, et al. Reactive arthritis induced by intravesical BCG therapy for bladder cancer. BMJ case reports 2014;2014:http://www.ncbi.nlm.nih.gov/pubmed/24510697

12. Bernini, L., C. U. Manzini, D. Giuggioli, et al. Reactive arthritis induced by intravesical BCG therapy for bladder cancer: our clinical experience and systematic review of the literature. Autoimmunity reviews 2013;12:1150-

9. http://www.ncbi.nlm.nih.gov/pubmed/23816569 
13. Oddens, J., M. Brausi, R. Sylvester, et al. Final results of an EORTC-GU cancers group randomized study of maintenance bacillus Calmette-Guerin in intermediate- and highrisk Ta, T1 papillary carcinoma of the urinary bladder: one-third dose versus full dose and 1 year versus 3 years of maintenance. European urology 2013;63:462-

72. http://www.ncbi.nlm.nih.gov/pubmed/23141049

14. Ojea, A., J. L. Nogueira, E. Solsona, et al. A multicentre, randomised prospective trial comparing three intravesical adjuvant therapies for intermediate-risk superficial bladder cancer: low-dose bacillus Calmette-Guerin ( $27 \mathrm{mg}$ ) versus very low-dose bacillus Calmette-Guerin (13.5 mg) versus mitomycin C. European urology 2007;52:1398406. http://www.ncbi.nlm.nih.gov/pubmed/17485161

15. Astram, A., A. Khadijah, P. Yuri, et al. Effective dose and adverse effects of maintenance Bacillus Calmette-Gue'Rin in intermediate and high risk non-muscle invasive bladder cancer: a meta-analysis of randomized clinical trial. Acta medica Indonesiana 2014;46:298-307. http://www.ncbi.nlm.nih.gov/pubmed/25633546 